\title{
Tratamiento de la enfermedad de Parkinson con palidotomía y palido-talamotomía estereotáctica guiada por microelectrodos
}

\author{
C. Llumiguano; T. Dóczi e I. Baths
}

Department of Neurosurgery. University of Pécs. Hungary.

\section{Resumen}

Objetivo. Los autores valoraron el efecto terapéutico de palidotomía y palido-talamotomía combinada guiada por MR y microelectrodos en 33 pacientes con enfermedad de Parkinson, cuyos síntomas fueron resistentes al tratamiento farmacológico.

Material y métodos. Los pacientes fueron evaluados en base a los test estandarizados a nivel internacional que se efectuaron antes y después de la operación: al $2^{\circ}$ día, así como a lo largo de 12 meses, con un intervalo de 3 meses entre ellos. Los pacientes fueron divididos en dos grupos; en los del grupo "A", los síntomas parkinsonianos, incluso el temblor, disminuyeron después de una palidotomía. En los pacientes del grupo "B", el temblor no disminuyó tras la palidotomía, razón por la cual ésta se complementó con una talamotomía.

Resultados. Con la ayuda de la escala UPDRS III se obtuvieron los siguientes resultados: después de la palidotomía en "fase on" el promedio preoperatorio de 51,2 disminuyó a 29,5 al $2^{\circ}$ día, a 26 a los 3, 6 y 9 meses, y a 28,7 a los 12 meses después de la operación; en "fase off" el promedio preoperatorio de 64,3 declinó a 31,6 al $2^{\circ}$ día, a 26 a los 3, 6 y 9 meses, y a 30,5 a los 12 meses después de la operación. Después de la palidotalamotomía en "fase on" el promedio preoperatorio de 43,5 disminuyó a 27,9 al $2^{\circ}$ día, a 22,9 a los 3 meses, a 22,8 a los 6 meses, y a 24,5 a los 9 y 12 meses después de la operación. De igual manera, en "fase off" el promedio preoperatorio de 62,6 declinó a 38 al $2^{\circ}$ día, a 30 a los 3 meses, a 31,8 a los 6 meses, y a 33,8 a los 9 y 12 meses después de la operación.

Conclusión. Para aquellos pacientes, en quienes la palidotomía no fue suficiente en el control del temblor, la palido-talamotomía fue efectiva. Los síntomas clínicos, de acuerdo con las escalas utilizadas, mejoraron significativamente en los dos grupos (student t:

Recibido: 10-05-05. Aceptado: 29-08-05
$\mathbf{P}<\mathbf{0 , 0 0 0 1 )}$; sin embargo, las lesiones bilaterales conllevan un alto riesgo de morbilidad.

PALABRAS CLAVE: Enfermedad de Parkinson. Palidotomía. Talamotomía.

Microeletrode guided stereotactic pallidotomy and pallido-thalamotomy for treatment of Parkinson's disease

\section{Abstract}

Objective. Authors evaluated the therapeutic effect of the MRI and microelectrodeguided stereotactic pallido- and pallido-thalamotomy in 33 patients with Parkinson's disease (PD), whose symptoms were refractory to pharmacological therapy.

Matherial and methods. The patients were evaluated according to the internationally standardized rating scales (UPDRS part II, III, Schawb \& England, Hoehn \& Yahr, and Fahn) at six timepoints: before the operation, and 2 days, 3, 6, 9 and 12 months postoperatively. The patients were divided into 2 groups. Those in group A had relief of all main parkinsonian symptoms after pallidotomy including tremor. The patients in group B had no relief of tremor after pallidotomy. For them the pallidotomy was completed by thalamotomy in the same sitting, which had resulted in cessation of tremor.

Results. The following results were obtained by using the UPDRS part III: after pallidotomy "On state" mean: preoperative 51,2 , postoperative at $2^{\text {nd }}$ day 29,5 at 3,6 and $9^{\text {th }}$ month 26 , and at $12^{\text {th }}$ month 28,7 . "Off state" mean: preoperative 64,3 , postoperative at $2^{\text {nd }}$ day

Abreviaturas. CRW: Cosman Robert Wells. GABA: Acido gamaamino butírico. Gpi: Globo pálido interno. Gpii: Globo pálido interno (segmento interno). Gpie: Globo pálido interno (segmento externo). Gpe: Globo pálido externo. MPTP: 1-methyl-1,2,36 tetra hydropiridina. PPV: Palidotomía postero-ventral. UPDRS: Unified Parkinson's Disease Rating Scale. Vim: Núcleo ventral intermedio del tálamo. Vop: Núcleo oral ventral posterior. 
31,6 , at 3,6 and $9^{\text {th }}$ month 26 , and at $12^{\text {th }}$ months 30,5 . After pallidothalamotomy "On state" mean: preoperative 43,5 , postoperative at $2^{\text {nd }}$ day 27,9 , at $3^{\text {rd }}$ month 22,9 , at $6^{\text {th }}$ month 22,8 , and at 9 and $12^{\text {th }}$ month 24,5 . "Off state" mean: preoperative 62,6, postoperative at $2^{\text {nd }}$ day 38 , at $3^{\text {rd }}$ month 30 , at $6^{\text {th }}$ month 31,8 and at 9 and $12^{\text {th }}$ month 33,8 .

Conclusions. For those patients, whose tremor was not successfully controlled by pallidotomy, the combined pallido-thalamotomy was effective. The clinical symptomps, according to the rating scales, improved significantly in both groups (student $t: P<0,0001$ ), but bilateral lesioning carried higher surgical morbidity.

KEY WORDS: Pallidotomy. Parkinson's disease. Thalamotomy.

\section{Introducción}

La enfermedad de Parkinson tiene un carácter progresivo y degenerativo, debido a la insuficiencia de dopamina en el cuerpo estriado y otros núcleos de los ganglios basales. Esto se debe a la ausencia de células dopaminérgicas en la parte compacta de la substancia negra ${ }^{12,23,36}$. Los principales síntomas de la enfermedad son: temblor, rigidez, bradicinesia, e inestabilidad postural $1^{4,22,23}$.

En la enfermedad de Parkinson, el tratamiento farmacológico se encuentra en primer lugar; sin embargo, en muchas circunstancias es necesaria la intervención quirúrgica, debido a que: 1) el tratamiento farmacológico, después de cierto tiempo, se vuelve insuficiente. 2) Las modernas técnicas con el avance de la monitorización neurofisiológica, han hecho posible que la neurocirugía funcional sea mucho más efectiva y segura. 3) El conocimiento anatómico, funcional y fisiopatológico de los ganglios basales, así como los experimentos en primates tratados con MPTP (1-methyl-4-phenyl-1,2,3,6-tetrahydropiridin) y las observaciones realizadas durante los mismos contribuyeron con valiosos datos científicos, a perfeccionar el tratamiento quirúrgico ${ }^{6,12,15,30,32}$.

Los síntomas inicialmente responden aceptablemente al tratamiento farmacológico. Sin embargo, tras cierto tiempo, el progreso de la enfermedad, así como el prolongado tratamiento farmacológico (L-dopa), disminuyen la efectividad del mismo ${ }^{10,23,43}$. Esto da lugar a una importante fluctuación de la actividad motora, desde el denominado estadío "on" (relativo establecimiento de las funciones motoras) hasta un estado de incapacitación total, (también conocido como estadío "off") que, incluso, llega hasta estadíos acinéticos/rígidos. Inicialmente, durante el estadío "off" se incrementan el tiempo de duración y la gravedad de los síntomas; junto a ellos aparecen otros síntomas producto del tratamiento farmacológico como las discinesias, que pueden variar desde una distonía lenta en forma de torsiones hasta movimientos rápidos y ásperos similares a los observados en una corea ${ }^{7,9,19,24}$. Estos movimientos pueden presentarse inmediatamente después de tomar los medicamentos, durante los niveles máximos, o durante la disminución de los niveles de concentración del medicamento. Además, los fármacos antiparkinsonianos pueden ocasionar efectos secundarios psíquicos, cognitivos, así como también molestias gastrointestinales, parecidos a aquellos ocasionados por la enfermedad misma. Cuando el paciente llega a este estadío indiscutiblemente necesita otras opciones terapéuticas.

\section{Fisiología de la palidotomía}

La substancia negra-pars reticulata, conjuntamente con el globo pálido (segmento interno) (Gpi) proveen una vía de salida de impulsos desde los ganglios basales. En pacientes de Parkinson, debido a la falta de dopamina, a través del sistema GABA se incrementa la activad inhibidora del Gpi. Según nuestros conocimientos actuales intervienen dos factores importantes: 1) Los sistemas excitatorios glutamatérgicos provenientes del núcleo subtalámico, que incrementan su actividad. 2) La disminución de la actividad inhibidora proveniente del estriado motor. La hiperactividad del Gpi ejerce una inhibición activa sobre el tálamo y el sistema descendente córtico-motor, el mismo que conduce a la aparición de los síntomas parkinsoniano $\mathrm{s}^{7,14,18,22,23,28,38}$. Por ello, el objetivo primordial de la cirugía es ocasionar una lesión selectiva en el Gpi para disminuir las alteraciones del sistema motor. Con objeto de eludir los efectos secundarios, la lesión debe respetar las regiones límbicas y asociativas del Gpi; de igual manera, no debe afectar al Gpi externo (Gpe), así como a las estructuras de vital importancia ubicadas a su alrededor, tales como la cápsula interna y el tracto óptico ${ }^{1,8,13,21,23,29,40}$.

\section{Indicación de la intervención}

En pacientes en quienes el temblor es el síntoma relevante, el objetivo quirúrgico más adecuado es el núcleo ventral-intermedio del tálamo (Vim), cuya lesión y estimulación eléctrica disminuyen los síntomas de forma eficaz $z^{3,411,17,37,39}$. Debemos considerar, además, que la palidotomía es capaz de aliviar parcialmente el temblor. Sin embargo, en muy raras circunstancias se presenta esta enfermedad con síntomas absolutamente tremorígenos. Casi siempre se dan síndromes parkinsonianos acinéticos/ rígidos, con fluctuación on/off, así como discinesias ocasionadas por el tratamiento farmacológico; para este último grupo de pacientes la palidotomía parece ser el tratamiento quirúrgico más adecuado.

Requisitos para la intervención: 1) enfermedad de 
Tabla 1

Concentración de la muestra

\begin{tabular}{|l|l|l|}
\hline Palidotomía & Unilateral - 6 & Bilateral - 7 \\
\cline { 2 - 3 } $\begin{array}{l}\text { Sexo } \\
\text { Edad promedio }\end{array}$ & Masculino - 6 & Femenino - 7 \\
\cline { 2 - 3 } Palido-talamotomía & 60,1 años & 5,4 años \\
\cline { 2 - 3 } $\begin{array}{l}\text { Sexo } \\
\text { Edad promedio }\end{array}$ & Unilateral - 10 & Bilateral - 10 \\
\cline { 2 - 3 } & Masculino - 14 & Femenino - 6 \\
\hline
\end{tabular}

Parkinson de carácter idiopático, 2) edad joven, 3) que los síntomas de la enfermedad reaccionen adecuadamente al tratamiento farmacológico inicial con L-dopa, los mismos que después de un tiempo se agravan, 4) presencia de efectos secundarios producto del tratamiento medicamentoso, tales como discinesia, rigidez, temblor, bradicinesia y alteraciones de la marcha y la postura, 5) función cognitiva intacta ${ }^{37,39,40}$. Hay que tener en cuenta que la enfermedad de Parkinson es un cuadro progresivo. La pálido-, y/o talamotomía es capaz de disminuir significativamente los síntomas, pero no se trata de una solución curativa ante la enfermedad, razón por la cual es imprescindible seguir con el tratamiento farmacológico, después de la intervención.

\section{Material y métodos}

Durante 3 años se realizaron en 13 pacientes 20 palidotomías (en 7 pacientes bilateral), y en 20 pacientes palidotalamotomía combinada, (bilateral en 10 de ellos). La distribución por sexos, en el caso de palidotomía, fue: sexo femenino 7, sexo masculino 6; en el caso de las intervenciones combinadas: sexo femenino 6, sexo masculino 14 . El tiempo transcurrido desde la aparición de los síntomas hasta el momento de la operación fue de 5,9 años; mientras que la edad promedio de los enfermos fue de 58,8 años. En 17 casos, debido a la presencia bilateral de los síntomas, se realizaron intervenciones bilaterales (Tabla 1).

De acuerdo con la sintomatología, los pacientes fueron divididos en dos grupos: a) aquéllos que se caracterizaron principalmente por presentar acinesia, rigidez, fluctuación on/off y discinesia inducida por L-dopa. La edad media de estos enfermos fue de 60,14 años y el tiempo medio desde la presentación de los síntomas al momento de la operación fue 6,5 años, b) los que, además de los síntomas antes mencionados, presentaron temblor en sus extremidades. En este grupo la edad media, fue 57,6 años y el tiempo medio transcurrido desde la aparición de los síntomas hasta el momento de la operación fue de 6,5 años. Teniendo en cuenta la sintomatología presentada, así como los diferentes datos bibliográficos, en aquellos pacientes del grupo "a" se efectuó una palidotomía, mientras que en los del grupo "b", en quienes la palidotomía no disminuyó el temblor, se complementó con una talamotomía.

\section{Valoracion clínica}

1.) La disminución del temblor fue valorada según la escala modificada de Fahn, la misma que permite una valoración cuantitativa; además, sirve para obtener una valoración funcional de la calidad de vida de los enfermos (escritura, valoración de los miembros superior e inferior, alimentación, higiene, vestimenta, marcha, capacidad de trabajo etc..). 2.) Los pacientes fueron valorados también con la escala modificada de Hoehn y Yahr en la fase on/off. 3.) Además se aplicó la escala para la valoración de los enfermos de Parkinson (UPDRS) : a) valoración de las actividades de la vida diaria (UPDRS II -ADL) y b) valoración de la actividad motora (UPDRS III - ME). 4.) Se añadió la escala modificada de Schwab y England (Modified Schwab \& England Activities of Daily Living Scale).

\section{Pasos de la operación}

\section{Aparatos}

Utilizamos el sistema estereotáctico de Radionics (Burhington, Massachusetts), que consta de un anillo craneal, al cual se adapta un marco de localización y un aparato de abordaje. El cálculo de las coordenadas del objetivo se realiza mediante un método algebraico directo RAD-SCS 1A-0494, en un ordenador con el programa Radionics. Finalmente colocamos en el anillo craneal el aparato de abordaje CRW (Cosman Robert Wells) teniendo en cuenta las tres coordenadas del objetivo: planos anterior-posterior, medio-lateral, y vertical.

Durante la electroestimulación y lesión de los ganglios basales se utilizó un generador de radiofrecuencia; (CRW Radionics RFG-3C Graphics), al cual se le adaptaron los termoelectrodos (Radionics TM/1,6/4/250 monitores de temperatura).

\section{Determinación del objetivo con la ayuda de resonancia magnética (RM)}

En el atlas estereotáctico, se puede determinar en milímetros de exactitud la ubicación de cada uno de los núcleos de los ganglios basales. Antes de la intervención se realizaron exámenes con RM (Magnetom Impact 1,0 T - Siemens, Erlangen) en T1 y T2, en todos los pacientes, en los planos coronal, sagital y axial. El aspecto fundamental es el ajuste del plano intercomisural, el cual se obtiene en los tres planos del espacio. Los objetivos en las imágenes de la RM se señalaron paralelamente al plano intercomisural. En el lado contralateral a los síntomas en las tomas axiales del plano intercomisural, tanto en el núcleo ventro-lateral 
del tálamo (Vim - en sentido antero-posterior a 3/10 de la distancia intercomisural [a $7-8 \mathrm{~mm}$ de la comisura posterior en sentido anterior], lateralmente a $5 / 10$ de la distancia intercomisural, tomados desde la parte central del III ventriculo [11 - $13 \mathrm{~mm}]$, y verticalmente en el plano intercomisural), así como en el Gpi (en sentido antero-posterior a 2 - $3 \mathrm{~mm}$ por delante del punto medio intercomisural, lateralmente a $18-20 \mathrm{~mm}$ medidos desde la pared lateral del III ventriculo y verticalmente de 3 - $4 \mathrm{~mm}$ por debajo del plano intercomisural).

\section{Determinación del objetivo con la ayuda del "fantom"}

En el fantom se comprueba la exactitud de las imágenes ofecidas por la RM, que se confirman en el aparato estereotáctico.

\section{Determinación del objetivo con la ayuda del microelec- trodo}

El objetivo fundamental de este método es que, a través del registro de la actividad celular, podamos determinar la ubicación de las células patológicas que ocasionan la actividad "burst" (descarga), las cuales señalan el sitio óptimo para la lesión. Para el registro de la actividad multiunitaria, utilizamos microelectrodos de wolframio de 0,5 - 1 Ohm de impedancia, cuyo amplificador se ubica cerca del electrodo. Usamos amplificadores especiales de dos canales, uno de los cuales cuenta con un filtro interior fino. Generalmente registramos entre $300 \mathrm{~Hz}-5 \mathrm{KHz}$ con amplificaciones de más de diez mil veces. En pacientes que presentan temblor, en el miembro afectado se colocan sensores electromagnéticos de movimiento; la salida del mismo se amplifica más de veinte mil veces en el otro canal del amplificador dentro de las escalas de 0,3 - 100 Hz. Los dos canales son digitalizados con la ayuda de un ordenador IBM. La importancia de este método se basa en que el objetivo determinado de forma empírica, debido a las diferencias morfológicas craneales de cada individuo, se puede localizar con algunos milímetros de inexactitud. La determinación exacta del objetivo (sitio de lesión), evita una innecesaria extensión de la lesión, la cual puede ser causa de síntomas no deseados, o bien de mortalidad en algunos casos.

\section{Medición de la actividad multiunitaria}

Los objetivos empíricos determinados en las imágenes de RM se abordan con métodos estereotácticos y micromanipuladores, bajo anestesia local.

A $10 \mathrm{~mm}$ por delante de la sutura coronal, a $10-15 \mathrm{~mm}$ de la línea media en sentido lateral, a través de un orificio de $2,5 \mathrm{~mm}$ de diámetro se introduce un microelectrodo hasta $12 \mathrm{~mm}$ antes del objetivo elegido. Con la ayuda del microelectrodo se observa la actividad celular como señales eléctricas emanadas de las estructuras del ganglio basal.
El microelectrodo, con la ayuda de un micromanipulador, y con movimientos de avance de $1-2 \mathrm{~mm}$, se dirige hacia el objetivo. Dependiendo de la calidad del registro se abre otra posibilidad para determinar con exactitud el óptimo lugar para la lesión. Esto es particularmente importante, ya que la determinación empírica del objetivo puede variar en algunos milímetros en relación al objetivo determinado por métodos electrofisiológicos, lo cual llevaría innecesariamente a una electrocoagulación de mayor magnitud y en un sitio no deseado. Esto podría ocasionar una disminución en la efectividad del tratamiento, y/o un incremento de la morbilidad.

Para el registro de las multiunidades se utilizan microelectrodos de wolframio (Radionics CRW SME) de 0,5 - 1 Mohm. El amplificador se coloca cerca del electrodo. En caso necesario, la perturbación del sistema puede eliminarse colocando una red de acero alrededor del microelectrodo y amplificador.

\section{Determinación del objetivo con métodos de estimulación intraoperatoria}

Con el electrodo de estimulación, y a distintas alturas, estimulamos las estructuras supuestas, y observamos los efectos positivos o negativos; por ejemplo: inducción, supresión del temblor, disartria, etc, así como también los umbrales del estímulo que son necesarios para provocar los mismos. Antes de la termocoagulación, los estímulos se realizaron con una serie de descargas de 2, 50, y 100 $\mathrm{Hz}$ de frecuencia, con tensiones de 0,5 - 3,0 voltios. En cada uno de los casos, determinamos el umbral de estímulo correspondiente a las reacciones tanto deseadas, como no deseadas.

\section{Localización del objetivo con el test de lesiones reversibles}

En los objetivos seleccionados mediante métodos electrofisiológicos realizamos una lesión reversible a baja temperatura, generalmente a $45-50{ }^{\circ} \mathrm{C}$ durante 20 segundos, antes de la termocoagulación. Con ello se interrumpe temporalmente la actividad de un determinado núcleo del ganglio basal. En caso de observar efectos secundarios no deseados, la lesión final queda descartada a dicha altura. Si durante el proceso no se presentan síntomas de déficit del sistema nervioso, entonces es factible la lesión final.

\section{Lesión irreversible con termocoagulación por radiofre- cuencia}

La termocoagulación o lesión irreversible la efectuamos a $75^{\circ} \mathrm{C}$, durante 60 segundos. Durante la lesión final se observó un espectacular apaciguamiento o desaparición de los desórdenes patológicos del movimiento. Si durante el proceso se observara la presencia de cualquier síntoma no deseado, se interrumpe la realización de lesión o se efectúa ésta a temperaturas inferiores a lo anteriormente indicado, 


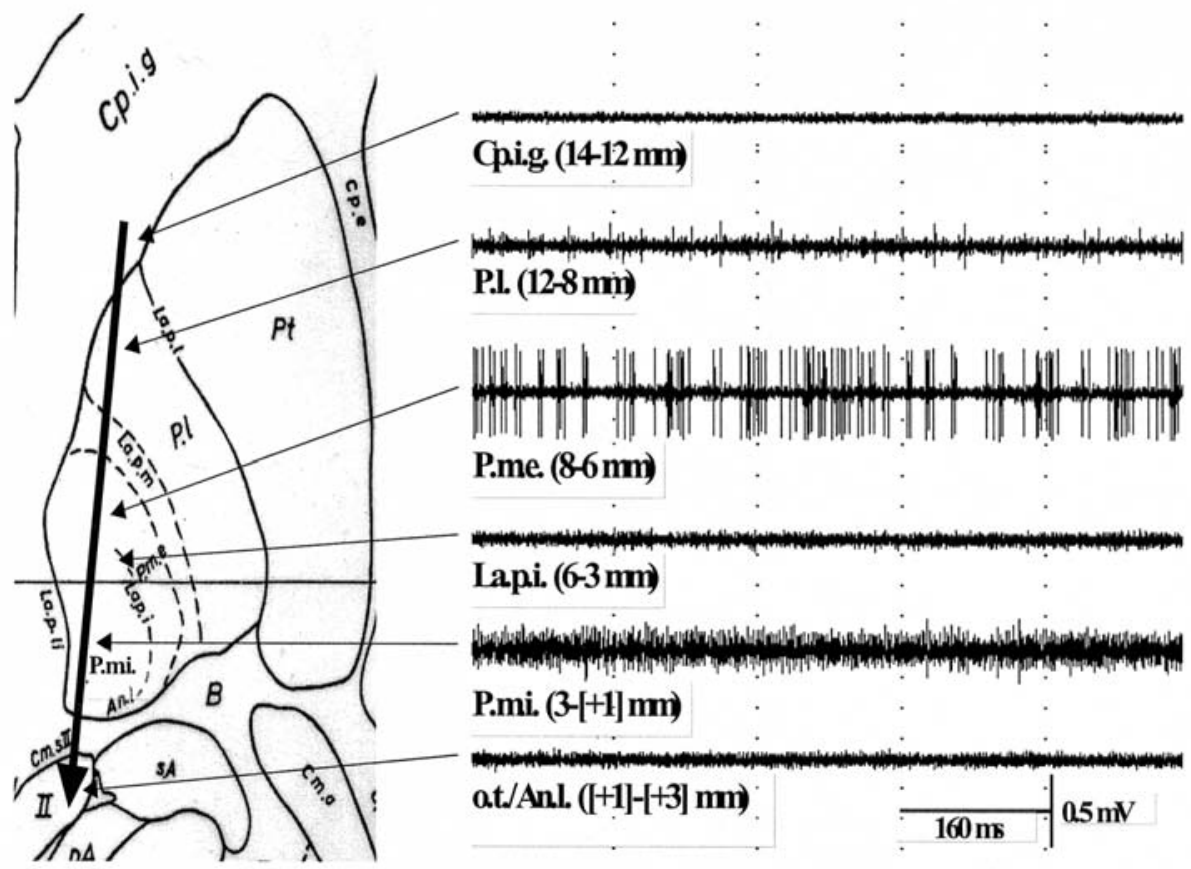

Figura 1. Lado izquierdo: Corte coronal según el atlas estereotáctico de Schaltenbrand a 2-3 mm de distancia del medio intercomisural en sentido craneal, y a 20-30 mm de distancia de la línea media del III ventrículo en sentido lateral. La flecha negra indica la orientación del microelectrodo sobre el Globus pallidus y lás estructuras circundantes. Abreviaturas: Cp.i.g.: cápsula interna genu. Cp.e.: cápsula externa. Pt.: putamen. La.p.1.: lamina pallidi lateralis, P.i.: pallidum laterale. La.p.m.: lámina pallidi mediale. P.m.e: pallidum mediale externum. La.p.i.: lamina pallidi incompleta. P.m.i.: pallidum mediale internum. La.p.li.: lamina pallidi limitans. II: tractus opticus. Cmc.II: chiasma opticum. An.1.: ansa lateralis. Lado derecho: registros de las multiunidades obtenidas con microelectrodo, indicando la distancia con respecto al blanco empírico.

teniendo en cuenta la disminución de los síntomas.

\section{Resultados}

\section{Actividad multiunitaria}

Las señales eléctricas obtenidas en cada unos de los pacientes examinados, de acuerdo con la distancia del objetivo se demuestran en un esquema gráfico (figura 1).

En el objetivo empírico Gpi o bien Cpi (a) 12 - 8 mm antes de llegar al mismo con la punta del electrodo, se observó un silencio eléctrico, es decir ausencia de actividad celular. Esto corresponde a la cápsula interna, debido a la presencia de substancia blanca, por lo que no se espera actividad celular alguna; (b) a 8 -6 mm del Gpi se observó actividad celular de gran amplitud y frecuencia, la misma que anatómicamente corresponde a la región del Gpe; (c) a $3 \mathrm{~mm}$ del Gpi, así como en el sitio del objetivo (target), y en algunas ocasiones a $1 \mathrm{~mm}$ más allá del objetivo y comparado con el registro anterior, se observó una actividad celular de baja amplitud pero alta frecuencia; este registro corresponde al Gpi. (d) finalmente, 1 - 3 mm sobrepasado el Gpi, se observó nuevamente un silencio eléctrico, en la zona que, según el atlas estereotáctico, corresponde al tracto óptico, así como también a la lámina de substancia blanca ubicada por debajo del Gpi.

\section{Palidotomía, en pacientes que no presentaron temblor}

Según la distribución por estadíos de Hoehn y Yahr en fase "on", el promedio preoperatorio de 3,42 descendió a un estadío de 2,85 , inmediatamente después de la operación, y a 2,87 a los 3, 6 y 9 meses, siendo de 3,13 de media 12 meses después de la operación. Igualmente en fase "of" de un promedio de 4,56 se pasó a un nivel de 2,83 inmediatamente después de la operación, a 2,87 a los 3, 6 y 9 meses, y a 3,13 a los 12 meses después de la operación (diagrama 1).

Según UPDRS - II. en fase "on" el promedio preoperatorio de 23,44 disminuyó hasta 12,5 puntos al segundo día de la operación, a 11,75 a los 3, 6 y 9 meses, y a 13,5 puntos a los 12 meses de la operación. De igual manera en fase "off" el promedio preoperatorio de 31,77 puntos bajó a 13,60 al $2^{\circ}$ día de la operación, a 11,75 a los 3, 6 y 9 meses y a 13,5 puntos a los 12 meses de la operación (diagrama 2).

Según UPDRS - III. en fase "on" el promedio preoperatorio de 51,22 puntos disminuyó hasta 29,50 al segundo día de la operación, a 26 a los 3, 6 y 9 meses, y a 


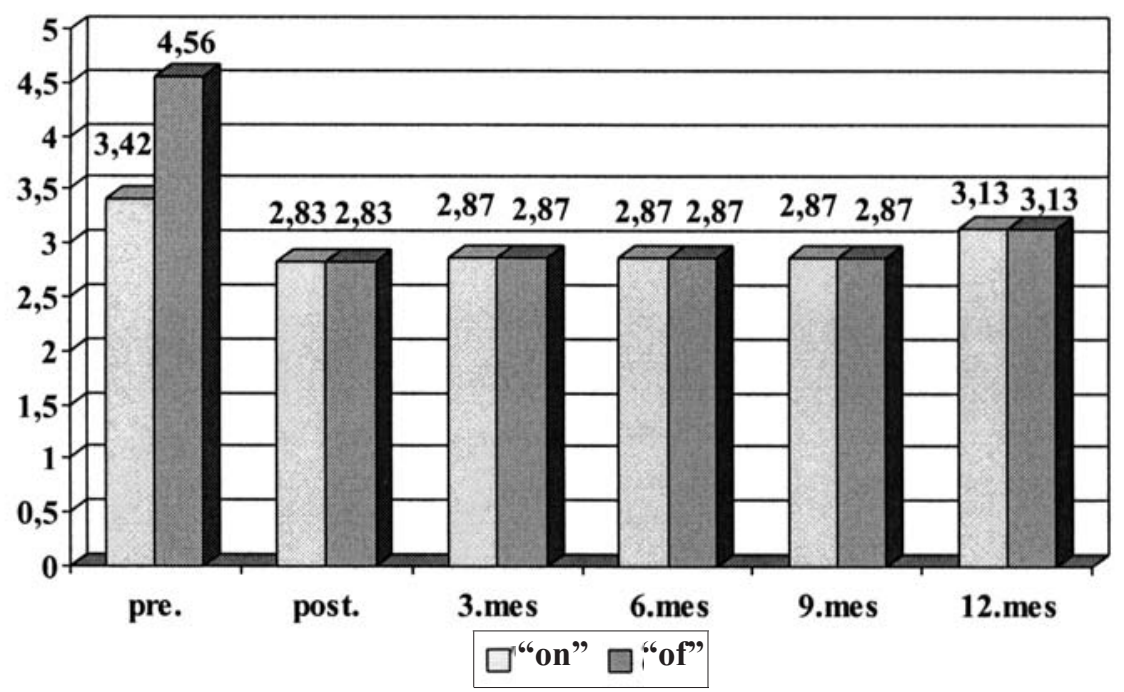

Diagrama 1. Escala modificada de Hg y Yahr, promedio de recuperación después de la palidotomía

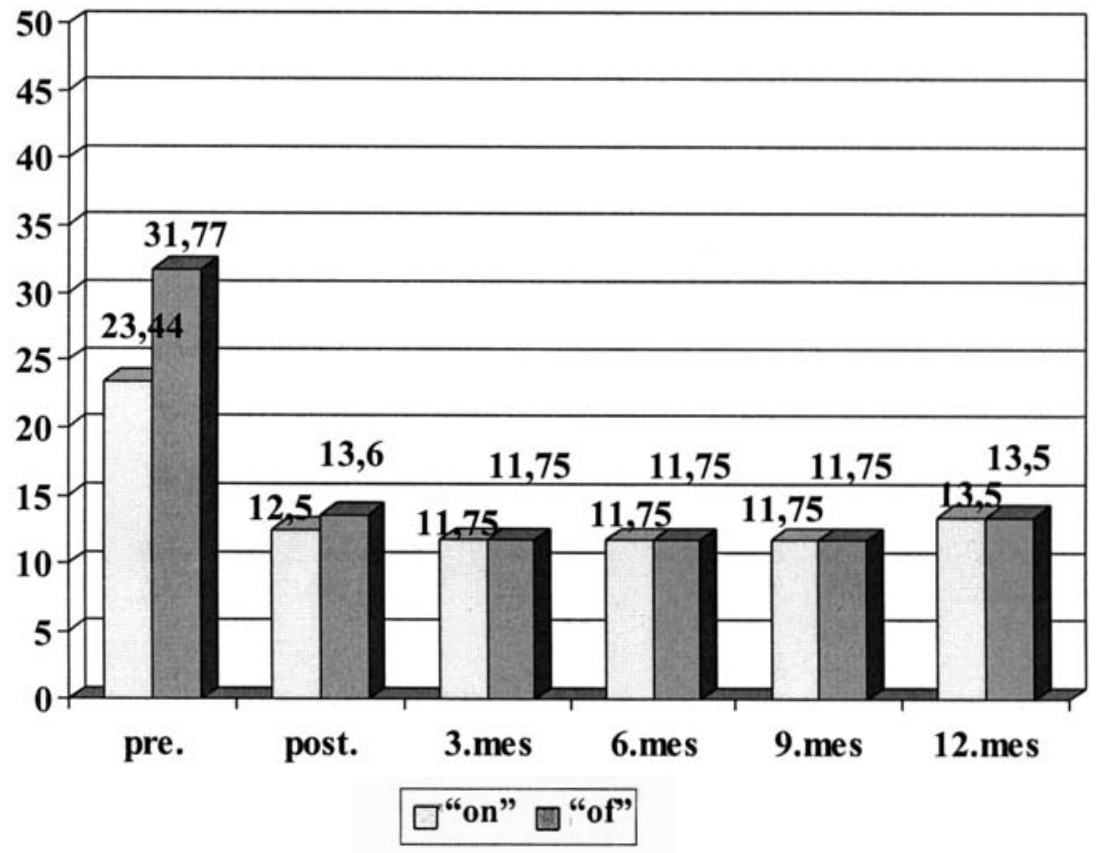

Diagrama 2. UPDRS II después de la palidotomía.

28,75 puntos a los 12 meses de la operación. En fase "off" el promedio preoperatorio de 64,33 puntos descendió a 31,60 al $2^{\circ}$ día de la operación, a 26 a los 3,6 y 9 meses y a 30,50 puntos a los 12 meses de la operación (diagrama 3).

Según la escala modificada de Schwab y England en la actividad diaria, el estado funcional en la fase "on" el promedio preoperatorio de $46 \%$ subió al $63 \%$ al segundo día de la operación, a $85 \%$ a los 3 y 6 meses, a $80 \%$ a los 9 meses, y al $72 \% 12$ meses después de la operación. De igual manera en la fase "off " el promedio preoperatorio de $13 \%$ subió a $63 \%$ al $2^{\circ}$ día de la operación, a $85 \% 3$ y 6 meses, a $80 \%$ a los 9 meses y a $72 \%$ a los 12 meses de la operación (diagrama 4).

Según las pruebas estadísticas "t", los resultados fueron significativos $(\mathrm{P}<0,0001)$.

Palido-talamotomía en pacientes que presentaron temblor

Según la escala modificada de Fahn, la puntuación 


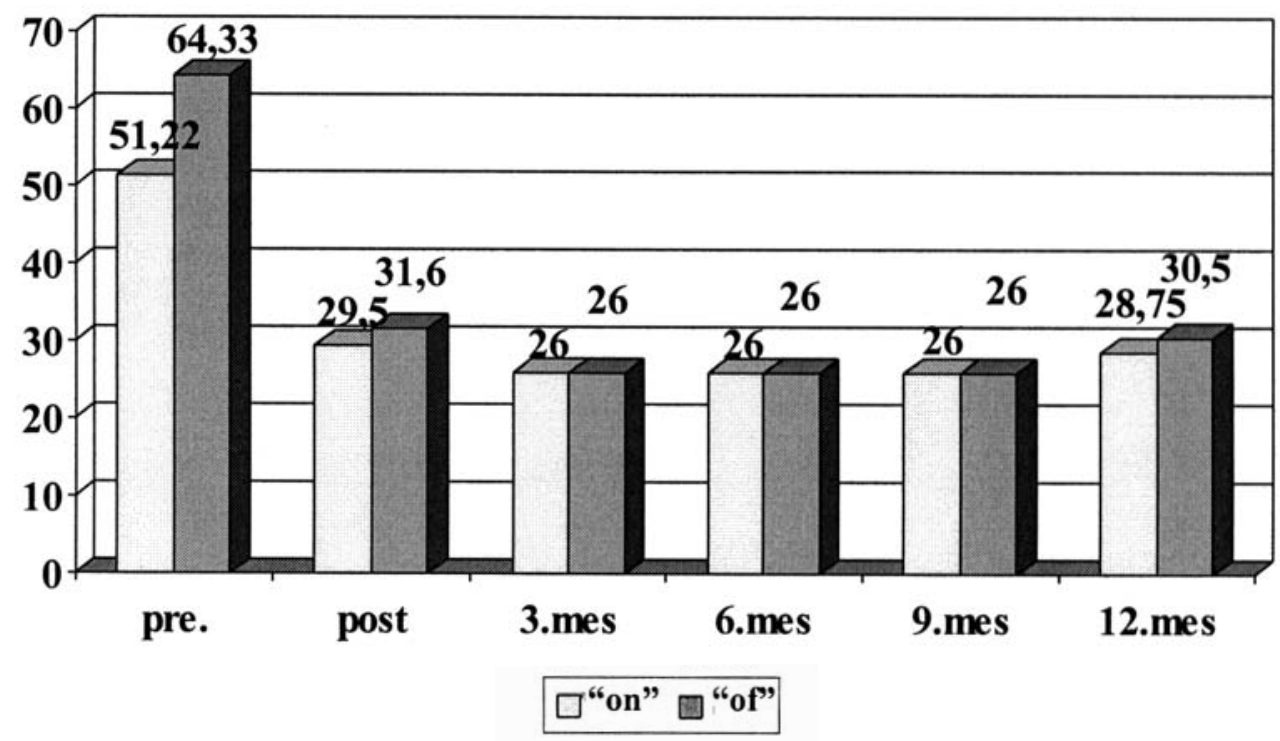

Diagrama 3. UPDRS III después de paÍidotomía

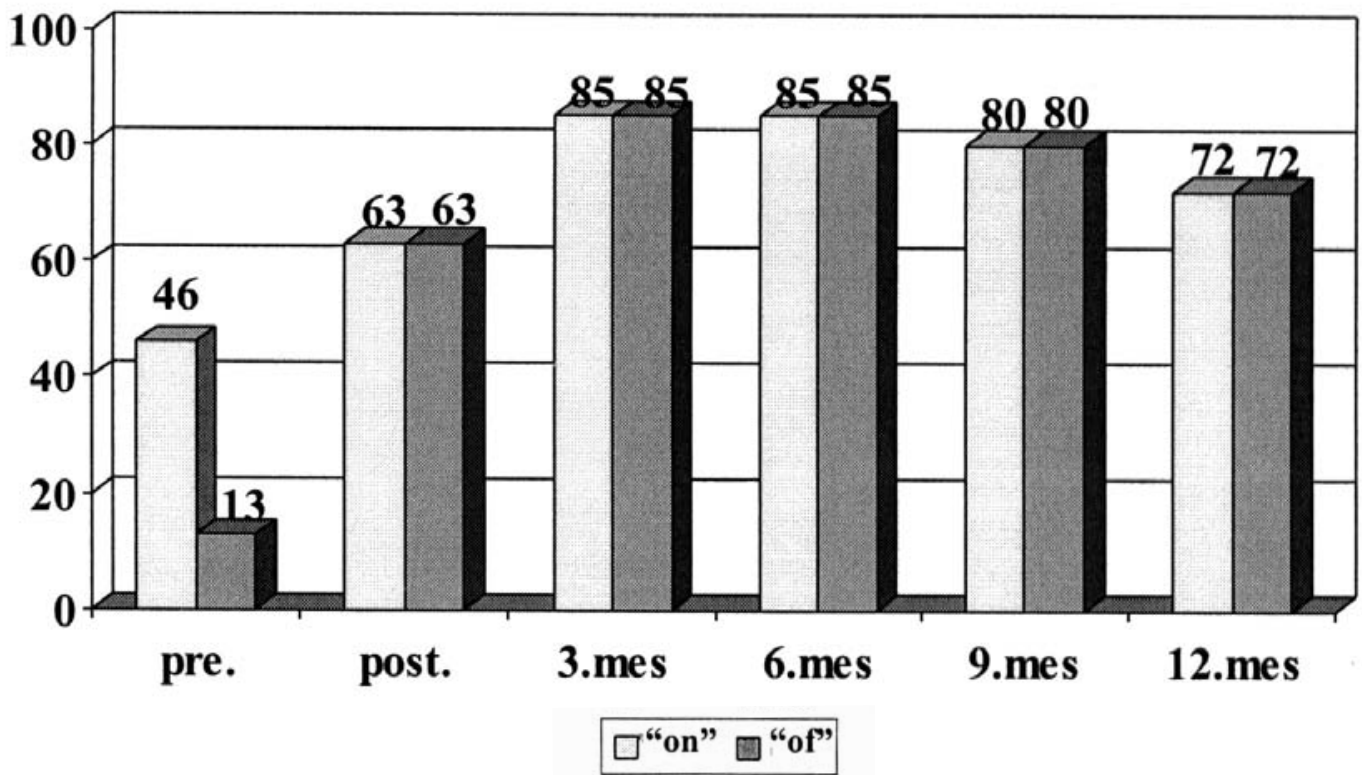

Diagrama 4. Promedio porcentual según la escala modificada de Schwab y England después de la palidotomía

preoperatoria promedio de 79,53 puntos bajó a 35,28 al $2^{\circ}$ día postoperatorio a 21,90 a los 3 meses, a 21,55 a los 6 meses, a 22,85 a los 9 meses, y a 22,71 puntos a los 12 meses de la operación (diagrama 5).

Según la distribución por estadíos de Hoehn y Yahr en fase "on" el promedio preoperatorio de 3,25 descendió a 1,62 inmediatamente después de la operación, a 1,95 a los 3 meses y a 2 a los 6,9 y 12 meses después de la operación. Igualmente en fase "off", de un promedio de 3,94 se pasó a un estadío de 1,72 inmediatamente después de la operación, a 2,83 a los 3 meses y a 3 a los 6,9 y 12 meses después de la operación (diagrama 6).
Según UPDS - II en fase "on" el promedio preoperatorio de 20,40 puntos disminuyó a 11,90 al $2^{\circ}$ día de la operación, a 11 a $\operatorname{los} 3$ meses, a 12,20 a los 6 meses y a 12,80 puntos a los 9 y 12 meses después de la operación. De igual manera en fase "off" el promedio preoperatorio de 28,20 puntos descendió hasta 7 al $2^{\circ}$ día de la operación, a 14,90 a los 3 meses, a 17,50 a los 6 meses ya 18,20 puntos a los 9 y 12 meses después de la operación (diagrama 7).

Según UPDRS - III. en fase "on" el promedio preoperatorio fue de 43,52 puntos, disminuyó hasta 27,90 al segundo día de la operación, a 22,90 a los 3 meses, a 22,80 a los 6 meses y a 24,50 a los 9 y 12 meses después 


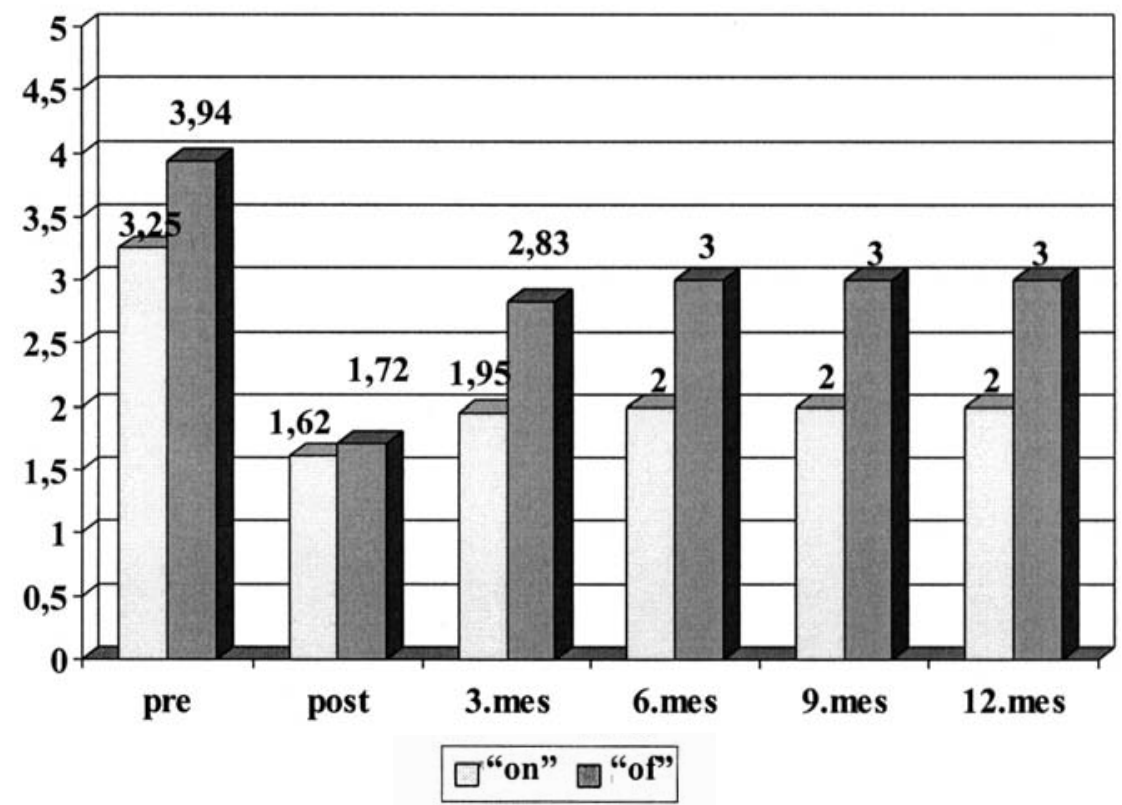

Diagrama 5. Promedio de recuperación, según la escala modificada de Fahn después de la palido-talamotomía combinada.

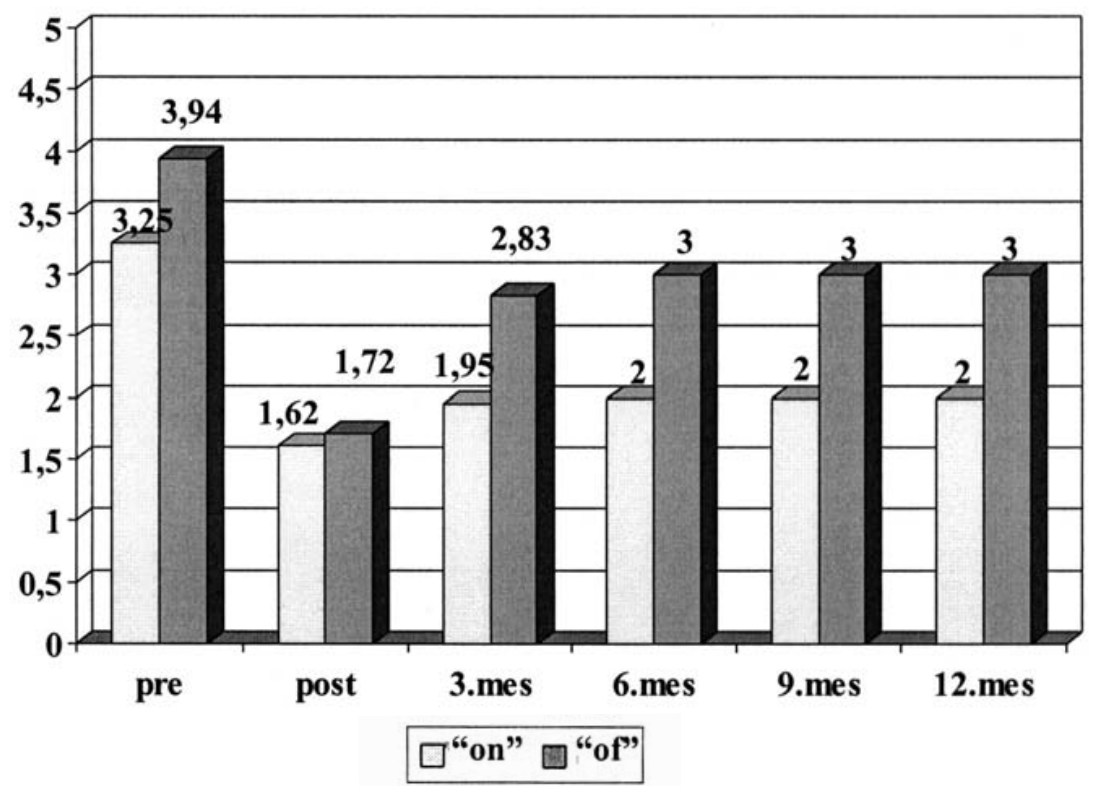

Diagrama 6. Promedio de recuperación, según la escala modificada de Hoehn y Yahr después de la palido-talamotomía combinada

de la operación. De igual manera, en fase "off" el promedio preoperatorio de 62,60 puntos bajó a 38 al $2^{\circ}$ día de la operación, a 30 a los 3 meses, a 31,83 a los 6 meses y a 33,80 puntos a los 9 y 12 meses después de la operación (diagrama 8).

Según la escala modificada de la actividad diaria de Schwab y England, en fase "on" el promedio preoperatorio de $57 \%$ subió a $79 \%$ al segundo día de la operación, a $83 \%$ a los 3 meses, a $79 \%$ a los 6 meses y a $78 \% 9$ y 12 meses después de la operación. De igual manera en fase "off” el promedio preoperatorio de $37 \%$ mejoró hasta el $70 \%$ al $2^{\circ}$ día de la operación, y hasta el $3^{\text {er }}$ mes y a los 6,9 y 12 meses después de la operación llegó al 64\% (diagrama 9).

Las pruebas estadísticas " $\mathrm{t}$ ", en todos los casos fue significativa $(\mathrm{P}<0,0001)$.

En el $88 \%(n=30)$ de los casos se logró alcanzar una supresión total o casi total del temblor, mientras que en el $12 \%(n=4)$ de los casos la supresión del temblor fue 


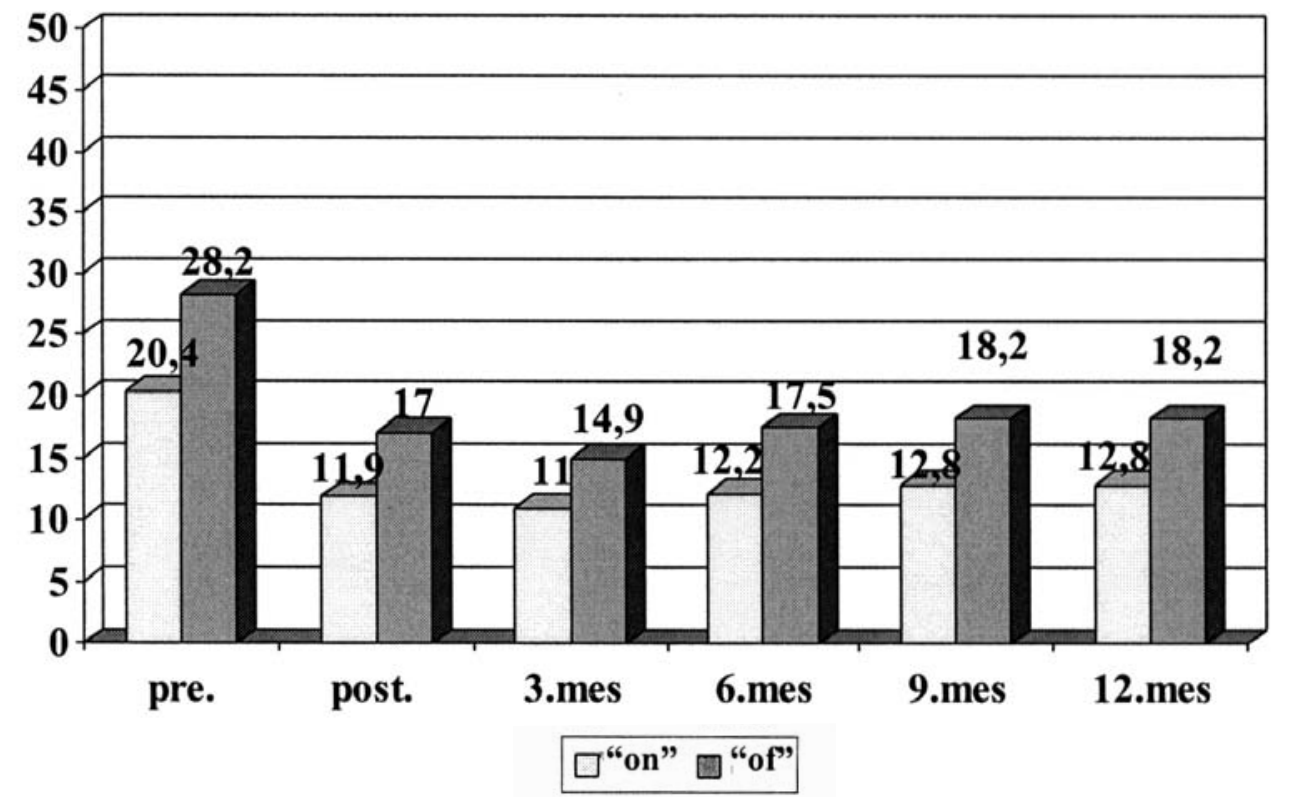

Diagrama 7. UPDRS II después de la palido-talamotomía combinada

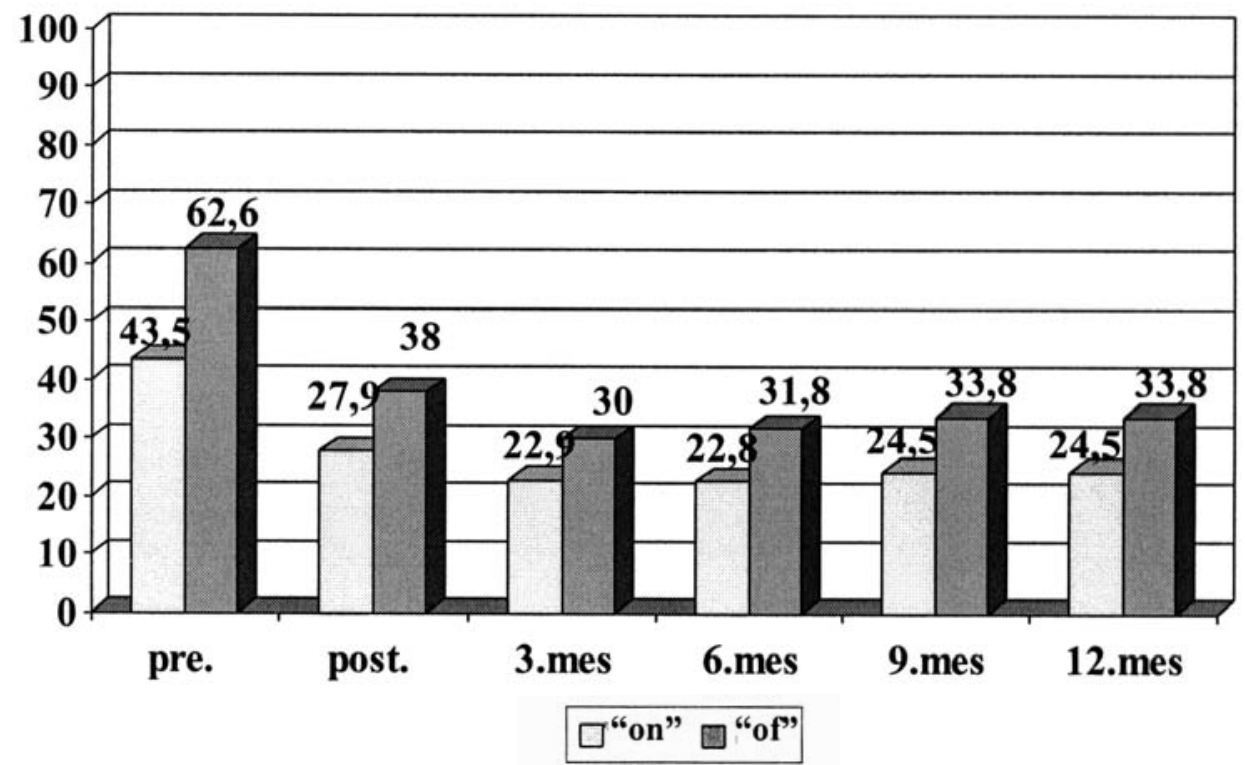

Diagrama 8. UPDRS III después de la palido-talamotomía combinada

sólo parcial.

\section{Complicaciones}

\section{Palidotomía}

Durante palidotomía bilateral en 1 paciente se observó disminución contralateral del campo visual temporal, como signo de lesión del tracto óptico.

\section{Palido-talamotomía combinada}

En el caso de operación unilateral, en 6 casos, se obser- varon complicaciones pasajeras mientras que en otros 3 casos las mismas tuvieron un carácter permanente. Cabe indicar que en las intervenciones bilaterales, las complicaciones, en 5 casos, fueron de tipo pasajero, en tanto que en 3 casos fueron permanentes, siendo éstas: disartia, alteraciones del equilibrio, hemiparesia latente y hemianopia.

No se presentaron casos de mortalidad postoperatoria.

\section{Discusión}

En este trabajo, se analizan en los dos grupos, los 


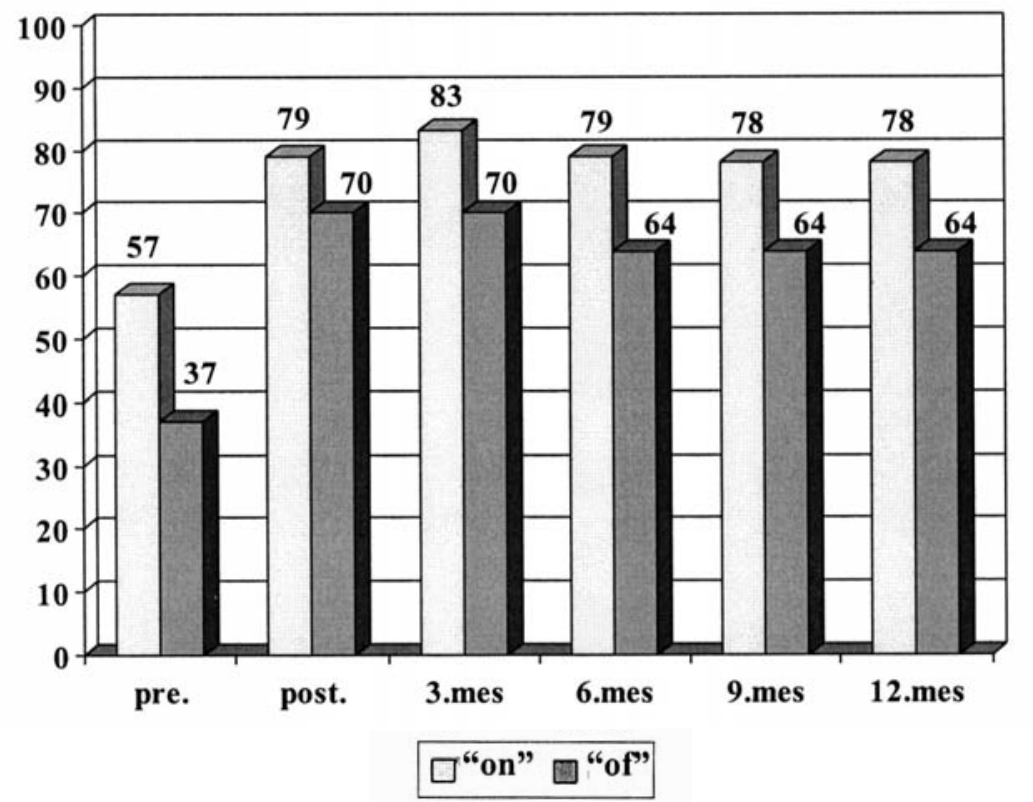

Diagrama 9. Promedio porcentual de recuperación, según la escala modificada de Schawb y England después de la palidotalamotomía combinada

resultados obtenidos por el efecto de la palidotomía, pues la talamotomía sólo se efectuó en casos graves de la enfermedad (pacientes, en quienes además de hipo-, bradicinesia y rigidez, también se detectó temblor en las extremidades), en los que el temblor no disminuyó después de la palidotomía. El objetivo fue analizar las ventajas que brinda la palidotomía, siempre y cuando los lugares de la lesión sean determinados mediante registro de la actividad celular patológica con ayuda del microelectrodo. Los estímulos a $2 \mathrm{~Hz}$ de frecuencia fueron para verificar la cercanía del tracto óptico y cápsula interna. Durante la estimulación del Vim talámico a $50 \mathrm{~Hz}$ de frecuencia, se observó inducción del temblor; sin embargo, en el caso de $100 \mathrm{~Hz}$ se observó una supresión del mismo en los miembros. En el caso del Gpi, a los $50-100 \mathrm{~Hz}$ de frecuencia se observó una disminución de rigidez y de la discinesia, así como una normalización del tono muscular. La cercanía a la cápsula interna durante la estimulación a 0,5 voltios de tensión y 2 $\mathrm{Hz}$ de frecuencia produjo la aparición de clonus en el miembro contralateral, mientras que el estímulo de 50 $100 \mathrm{~Hz}$ de frecuencia produjo contracción muscular tónica en los músculos de la mímica y en los miembros del lado contralateral.

Cuando el "target" determinado con métodos empíricos se localizó muy cerca de la cápsula interna, durante la estimulación se presentaron disartria a $50-100 \mathrm{~Hz}$, y clonus a $2 \mathrm{~Hz}$. Con $100 \mathrm{~Hz}$ de frecuencia se produjo contracción muscular tónica contralateral, tanto en los músculos de la cara, como en los miembros, aun por debajo de los 0,5 voltios de tensión. La presencia del tracto óptico o la cercanía al mismo se detectó con un umbral de estímulo de 0,1 - 0,5 voltios y $2 \mathrm{~Hz}$ de frecuencia, al tiempo que es advertida por el paciente como "visión de luces y estrellas".

De acuerdo con los datos bibliográficos, la palidotomía fue eficaz sobre la rigidez e hipocinesia en el $92 \%$ de los casos; el temblor disminuyó en el $81 \% \%^{5,7,10,17,20,22,31,32,37}$, lo cual concuerda con los resultados obtenidos durante nuestro trabajo. Varios autores se refieren a la importancia del uso de microelectrodos en el Gpi (región somatosensorial), ya que permite determinar el sitio óptimo para la lesión. Según ellos, es imprescindible el microrregistro para localizar las estructuras anatómicas ${ }^{13,21,25-27}$.

Es conocido que en el Gpi existen 2 vías eferentes principales, de las cuales la primera, inhibidora, se dirige hacía el tálamo y la corteza cerebral (palido-tálamo-cortical), y una segunda (inhibición GABA-érgica) hacia el núcleo pedunculopontino. Por otro lado, es de conocimiento general que, al contrario de la palidotomía, la talamotomía, en esencia, disminuye los síntomas hipercinéticos, como el temblor y la discinesia ${ }^{16,26,37}$.

En realidad, la palidotomía no suprime de forma eficaz el temblor como lo hace la talamotomía, razón por lo cual se introdujeron procedimientos quirúrgicos combinados (en el núcleo Vim, Ventral - oral - posterior /Vop/, del tálamo, así como en el globo pálido región postero ventral $/$ palidotomía postero-ventral $=\mathrm{PPV} /)^{17,19,32,38}$. La talamotomía (Vim) trae como resultado la interrupción del círculo rubro-tálamo-cortical, mientras que la palidotomía interrumpe la eferencia de las fibras del Gpi, lo cual podría interferir o bloquear el efecto inhibitorio del mesencéfalo y del centro locomotor espinal. Por este motivo, durante nuestro trabajo en los pacientes del grupo $\mathrm{B}$, además de 
la palidotomía nos vimos obligados a realizar talamotomías, con objeto de abolir o disminuir los movimientos hipercinéticos. Las intervenciones combinadas atenuaron de forma eficaz no solamente los síntomas hipercinéticos (temblor), sino también los síntomas hipocinéticos (rigidez, bradicinesia, alteraciones de la postura y la marcha). Sin embargo, hay que indicar que las intervenciones bilaterales conllevan un alto riesgo de morbilidad.

En nuestra publicación, nuestro deseo es hacer hincapié en la importancia de la determinación del blanco o diana con la ayuda de microelectrodos durante la operación. El blanco empírico, es decir, aquél señalado en las imágenes de RM concordantes con el atlas estereotáctico, nos permite un conocimiento real de la ubicación exacta del ganglio basal (Gpi), así como la topografía individual y la extensión del mismo. Con ayuda del microelectrodo se puede demostrar la actividad celular característica de cada uno de los 3 segmentos de la parte postero-ventral del globo pálido (Gpe, Gpi segmento interno = Gpii, Gpi segmento externo $=$ Gpie). Otro de los objetivos de nuestro trabajo fue mostrar el Gpi con microrregistros, así como los potenciales de acción de las estructuras anatómicas circundantes, lo cual nos ayuda en la determinación de objetivo final. Además, el uso del microelectrodo nos brinda la posibilidad de evitar lesionar importantes estructuras anatómicas, cercanas al objetivo (Gpi)) carentes de células nerviosas, tales como cápsula interna y el tracto óptico. El uso del microelectrodo incrementa de esta manera la importancia, efectividad y seguridad de la cirugía estereotáctica en la enfermedad de Parkinson.

Cabe indicar, además que, en nuestro trabajo, se detectó un bajo índice de complicaciones, tanto pasajeras, como permanentes, las cuales ,según la literatura, suelen presentarse de forma más frecuente ${ }^{1,2,7,10,17,32-35,37,41,42}$.

\section{Bibliografía}

1. Baron, M.S., Vitek, J.L., Bakay, R.A., Green, J., McDonald, W.M., Cole, S.A., et al.: Treatment of advanced Parkinson's disease by unilateral posterior GPi pallidotomy: 4year results of a pilot study. Mov Disord. 2000 ; 15: 230-237.

2. Bastian, A.J., Kelly, V.E., Perlmutter, J.S., Mink, J.W.: Effects of pallidotomy and levodopa on walking and reaching movements in Parkinson's disease. Mov Disord. 2003; 18: 1008-1017.

3. Brudzynski, S.M., Wu, M., Mogenson, G.J.: Decreases in rat locomotor activity as a result of changes in synaptic transmission to neurons within the mesencephalic locomotor region. Can J Physiol Pharmacol. 1993; 71: 394-406.

4. Burchiel, K.J.: Thalamotomy for movement disorders. Neurosurg Clin N Am. 1995; 6: 55-71.

5. Carr, J.A., Honey, C.R., Sinden, M., Phillips, A.G., Martzke, J.S.: A waitlist control-group study of cognitive, mood, and quality of life outcome after posteroventral pallidotomy in Parkinson disease. J Neurosurg. 2003; 99: 78-88.

6. Chabolla, D.R., Maraganore, D.M., Ahlskog, J.E., O'Brien, P.C., Rocca, W.A.: Drug-induced parkinsonism as a risk factor for Parkinson's disease: a historical cohort study in Olmsted County, Minnesota. Mayo Clin Proc. 1998; 73: 724727.

7. De Bie, R.M., Schuurman, P.R., Esselink, R.A., Bosch, D.A., Speelman, J.D.: Bilateral pallidotomy in Parkinson's disease: a retrospective study. Mov Disord. 2002; 17: 533538.

8. DeVito, J.L., Anderson, M.E.: An autoradiographic study of efferent connections of the globus pallidus in Macaca mulatta. Exp Brain Res. 1982; 46: 107-117.

9. Fazzini, E., Dogali, M., Sterio, D., Eidelberg, D., Beric, A.: Stereotactic pallidotomy for Parkinson's disease: a longterm follow-up of unilateral pallidotomy. Neurology. 1997; 48: 1273-1277.

10. Fine, J., Duff, J., Chen, R., Chir, B., Hutchison, W., Lozano, A.M., et al.: Long-term follow-up of unilateral pallidotomy in advanced Parkinson's disease. N Engl J Med. 2000; 342: 1708-1714.

11. Fox, M.V., Ahlskog, J.E., Kelly, P.J.: Stereotactic ventrolateralis thalamotomy for medically refractory tremor in post-levodopa era Parkinson's disease patients. J Neurosurg. 1991; 75: 723-730.

12. Garcia-Rill, E.: The basal ganglia and the locomotor regions. Brain Res. 1986; 396: 47-63.

13. Garonzik, I.M., Hua, S.E., Ohara, S., Lenz, F.A.: Intraoperative microelectrode and semi-microelectrode recording during the physiological localization of the thalamic nucleus ventral intermediate. Mov Disord. 2002; 17 Suppl 3: S135-44.

14. Gershanik, O.S.: Early onset parkinsonian. Front Biosci. 2003; 8: s568-78.

15. Guehl, D., Pessiglione, M., Francois, C., Yelnik, J., Hirsch, E.C., Feger, J., et al.: Tremor-related activity of neurons in the 'motor' thalamus: changes in firing rate and pattern in the MPTP vervet model of parkinsonism. Eur J Neurosci. 2003; 17: 2388-2400.

16. Hariz, G.M., Lindberg, M., Hariz, M.I., Bergenheim, A.T.: Does the ADL part of the unified Parkinson's disease rating scale measure ADL? An evaluation in patients after pallidotomy and thalamic deep brain stimulation. Mov Disord. 2003; 18: 373-381.

17. Hariz, G.M., Lindberg, M., Hariz, M.I., Bergenheim, A.T.: Gender differences in disability and health-related quality of life in patients with Parkinson's disease treated with stereotactic surgery. Acta Neurol Scand. 2003; 108: 28-37.

18. Hughes, A.J., Daniel, S.E., Ben-Shlomo, Y., Lees, A.J.: The accuracy of diagnosis of parkinsonian syndromes in a specialist movement disorder service. Brain. 2002; 125 : 861870. 
19. Iacono, R.P., Henderson, J.M., Lonser, R.R.: Combined stereotactic thalamotomy and posteroventral pallidotomy for Parkinson's disease. J Image Guid Surg. 1995; 1: 133-140.

20. Johansson, F., Maim, J., Nordh, E., Hariz, M.: Usefulness of pallidotomy in advanced Parkinson's disease. J Neurol Neurosurg Psychiatry. 1997; 62: 125-132.

21. Kopyov, O., Jacques, D., Duma, C., Buckwalter, G., Kopyov, A., Lieberman, A., et al.: Microelectrode-guided posteroventral medial radiofrequency pallidotomy for Parkinson's disease. J Neurosurg. 1997; 87: 52-59.

22. Lai, E.C., Jankovic, J., Krauss, J.K., Ondo, W.G., Grossman, R.G.: Long-term efficacy of posteroventral pallidotomy in the treatment of Parkinson's disease. Neurology. 2000; 55: 1218-1222.

23. Laitinen, L.V., Bergenheim, A.T., Hariz, M.I.: Leksell's posteroventral pallidotomy in the treatment of Parkinson's disease. J Neurosurg. 1992; 76: 53-61.

24. Laitinen, L.V.: Pallidotomy for Parkinson's disease. Neurosurg Clin N Am. 1995; 6: 105-112.

25. Lang, A.E., Lozano, A.M., Montgomery, E., Duff, J., Tasker, R., Hutchinson, W.: Posteroventral medial pallidotomy in advanced Parkinson's disease. N Engl J Med. 1997; 337: 1036-1042.

26. Lozano, A.M., Hutchison, W.D.: Microelectrode recordings in the pallidum. Mov Disord. 2002; 17 Suppl 3: S150154.

27. Molinuevo, J.L., Valldeoriola, F., Rumia, J., Nobbe, F.A., Ferrer, E., Tolosa, E.: Efficacy and safety of posteroventral pallidotomy for the treatment of advanced Parkinson's disease. Med Clin (Barc). 2000; 114: 205-208.

28. Obeso, J.A., Rodríguez, M.C., Gorospe, A., Guridi, J., Alvarez, L., Macias, R.: Surgical treatment of Parkinson's disease. Baillieres Clin Neurol. 1997; 6: 125-145.

29. Obeso, J.A., Guridi, J., Alvarez, L., Macias, R., Linasoro, G.: Ablative surgery for Parkinson's disease. In Jankovic J, Tolosa E, (eds). Parkinson's disease and movement disorders. 3.Ed. Media: Williams \& Wilkins, 1998: 1049-1064.

30. Ohye, C., Shibazaki, T.: Lesioning the thalamus for dyskinesia. Stereotact Funct Neurosurg. 2001; 77: 33-39.

31. Pal, P.K., Samii, A., Kishore, A., Schulzer, M., Mak, E., Yardley, S., et al.: Long term outcome of unilateral pallidotomy: follow up of 15 patients for 3 years. J Neurol Neurosurg Psychiatry. 2000; 69: 337-344.

32. Parkin, S.G., Gregory, R.P., Scott, R., Bain, P., Silburn, P., Hall, B., et al.: Unilateral and bilateral, pallidotomy for
Idiopathic Parkinson's disease: a case series of 115 patients. Mov Disord. 2002; 17: 682 -692 .

33. Roberta Rodrigues, R., Borges, V., Aguiar, P., Ferraz, F., Hisatugo, M., Ferraz, H.: Daily living activities in Parkinson's disease patients underwent to stereotactic surgery. Arq. Neuro-Psiquiatr. vol. 60 no. 2B Sao Paulo 2002.

34. Schrag, A., Samuel, M., Caputo, E., Scaravilli, T., Troyer, M., Marsden, et al.: Unilateral pallidotomy for Parkinson's disease: results after more than 1 year. J Neurol Neurosurg Psychiatry. 1999; 67: 511-517.

35. Shannon, K.M., Penn, R.D., Kroin, J.S., Adler, C.H., Janko, K.A., York, M., et al.: Stereotactic pallidotomy for the treatment of Parkinson's disease efficacy and adverse events at 6 months in 26 patients. Neurology. 1998; 50: 434-438.

36. Shima, F., Ishido, K., Sun, S.J., Machi, T., Kamikaseda, K., Fukui, M., et al.: Surgical control of akinesia in Parkinson's disease. Eur Neurol. 1996; 36 Suppl 1:55-61.

37. Sidiskis, J.: Indications for surgical treatment of Parkinson's disease and outcome. Medicina (Kaunas). 2003; 39: 990-995.

38. Slawek, J.: Stereotactic surgery in Parkinson disease: patient selection criteria in the light of existing research. Neurol Neurochir Pol. 2003; 37: 215-227.

39. Speelman, J.D., Schuurman, P.R., de Bie, R.M., Bosch, D.A.: Thalamic surgery and tremor. Mov Disord. 1998; 13 Supp13: 103-106.

40. Takeuchi, J., Yamagami, T., Saiki, H.: Details and indications of pallidotomy and thalamotomy for Parkinson's disease. Nippon Rinsho. 2000; 58: 2072-2077.

41. Uitti, R.J.: Surgical treatments for Parkinson's disease. Can Fam Physician. 2000; 46: 368-373.

42. Valldeoriola, F., Martínez-Rodríguez, J., Tolosa, .E, Rumia, J., Alegret, M., Pilleri, M., et al.: Four year followup study after unilateral pallidotomy in advanced Parkinson's disease. J Neurol. 2002; 249: 1671-1677.

43. Vidailhet, M.: Heterogeneity of Parkinson's disease. Bull Acad Natl Med. 2003; 187: 259-275.

Llumiguano, C.; Dóczi, T.; Baths, I.: Tratamiento de la enfermedad de Parkinson con palidotomía y palidotalamotomía estereotáctica guiada por microelectrodos. Neurocirugía 2006; 17: 420-432.

Correspondencia postal: Dr. Carlos Llumiguano M.D. Department of Neurosurgery. University of Pécs. H-7623, Pécs, Rét utca 2 sz. Hungary. 
Comentario al trabajo Tratamiento de la enfermedad de Parkinson con palidotomía y palido-talamotomía estereotáctica guiada por microelectrodos de C. Llumiguano y cols.

Los autores presentan los resultados en una serie de 33 pacientes con enfermedad de Parkinson que fueron tratados con palidotomía (13 pacientes) o palidotalamotomía combinada (20 pacientes).

Suponemos que los autores optaron por la palidomía o palido-talamotomía como técnica terapéutica de elección debido a la no disponibilidad de técnicas de estimulación cerebral profunda ya que éstas parecen mostrar los mismos beneficios clínicos pero con un menor número de efectos secundarios ${ }^{2}$. El punto diana óptimo dentro del GPi sigue siendo debatido y los resultados de la palidotomía son muy variables entre las series dependiendo en gran modo de los criterios de inclusión, técnica empleada, y si el procedimiento se realiza uni o bilateralmente ${ }^{4}$. En la serie presentada el número de complicaciones es mayor al reportado por otros autores ${ }^{3}$, especialmente en pacientes con palido-talamotomía. De los 10 pacientes en los que se realizó este procedimiento unilateralmente, 6 presentaron complicaciones pasajeras y 3 permanentes y en los otros 10 pacientes en los que se realizó una palido-talamotomía bilateral, 5 presentaron complicaciones pasajeras y 3 permanentes. Sin embargo, es interesante que la incidencia de complicaciones sea similar en los procedimientos uni o bilaterales, lo que contradice la observación de Alterman y Kelly quienes creían que los procedimientos bilaterales sobre el pálido jugaban un pobre papel en el tratamiento de la enfermedad de Parkinson dado su limitada utilidad clínica y al alta incidencia de "speech arrest"1 a pesar de realizar el procedimiento quirúrgico de forma estadiada.

Por último, los resultados reportados pueden llevar a confusión ya que la palidotalamotomía no fue una opción terapéutica de primera elección sino un tratamiento "de rescate" en aquellos pacientes en los que la palidotomía aislada resultó fallida. Así los resultados del grupo A (13 pacientes) representan sólo y exclusivamente a aquellos pacientes con una respuesta positiva a la palidotomía como tratamiento inicial y no al conjunto de pacientes sometidos a este procedimiento. Para evaluar, en esta serie, la respuesta real de los pacientes parkinsonianos sometidos a palidotomía, los resultados iniciales previos a la realización de la talamotomía del Grupo B deberían ser incluidos ("palidotomía primaria ineficaz"). Sin embargo, esto no puede ser analizado ya que al parecer, la decisión de realizar la talamotomía adicional se realizó durante el mismo acto quirúrgico.

\section{Bibliografía}

1. Alterman, R.L., Kelly, P.J.: Pallidotomy technique and results: the new New York University experience. Neurosurg Clin North Am 1998; 9: 337-343.

2. Krack, P., Pollak, P., Limousin, P., Hoffmann, D., Xie, J., Benazzouz, A., Benabid, A.L.: Subthalamic or internal pallidal stimulation in young onset Parkinson's disease. Brain 1998; 121: 451-457.

3. Laitinen, L.V., Bergenheim, A.T., Hariz, M.I.: Ventroposterolateral pallidotomy can abolish all parkinsonian symptoms. Stereotac Funct Neurosurg 1992; 58: 14-21.

4. Payne, B.R.,. Bakay, R.A.E., Vitek, J.K.: Pallidotomy for treatment of Parkinson's disease. In: Krauss JK, Jankovic J y Grossman RG eds. Surgery for Parkinson's disease and movement disorders. Philadelphia: Lippincott William and Wilkins 2001; 161-169.
P. Miranda Lloret R. Alday Anzola Madrid 